Ann. Biol. anim. Bioch. Biophys., 1977, 17 (3 B), 403-412.

\title{
Les cellules à prolactine chez la Ratte gestante, le fotus, le nouveau-né et le jeune.
}

\author{
par A. CHATELAIN *, M. P. DUBOIS **, J. P. DUPOUY * \\ avec la collaboration technique de J. P. POZZO DI BORGO et J. B. ZAZAC \\ * Laboratoire de Physiologie Animale, Faculté des Sciences. \\ 33, rue St-Leu, 80039 Amiens Cedex \\ ** Laboratoire de Neuroendocrinologie sexuelle, I.N.R.A., \\ Nouzilly, 37380 Monnaie
}

Summary. Prolactin cells in pregnant rats, rat fetuses and neonates and suckling rats.

Prolactin cells are studied in pituitary sections of female rats from day 15 to day 21 of pregnancy, in rat fetuses and neonates and in suckling rats using unlabelled rat prolactin antibody and the soluble peroxidase-antiperoxidase complex.

In the maternal pituitary, many cells react with the specific antibody in the pars distalis at all the gestational stages studied. The cytoimmunological reaction reaches a maximum on days 20 and 21.

In the fetal hypophysis, the first prolactin cells are found on day 21 of pregnancy located near the pituitary cleft and in the posterior part of the pars disfalis ; reaction intensity is very weak.

Pituitary sections from 1 to 4-day old neonates contain as few prolactin cells as those of 21-day old fetuses. From day 6 to day 20 post partum, the number and intensity of reactive cells increases. From day 8 on, the pituitary sections of the females contain more reactive cells than those of the males.

To investigate whether the fetal hypothalamus is involved in the cytodifferentiation of the prolactin cells, rat fetuses are encephalectomized in ufero on day 16 of pregnancy. Pituitary sections from encephalectomized and normal littermate fetuses are studied on day 21. The cells revealed with rat prolactin antibody are as numerous in the pars disfalis of the encephalectomized fetuses as in the controls.

These data suggest that the cytodifferentiation of rat hypophysis prolactin cells is not under hypothalamic control.

\section{Introduction.}

Chez la ratte gestante, la teneur du plasma en prolactine s'élève brutalement au cours des 2 ou 3 jours qui précèdent la parturition (Amenomori et al., 1970 ; Bast et Melampy, 1972, Linkie et Niswender, 1972 ; Nagasawa et Yanai, 1972 ; Morishige et al. 1973 ; Shiu et al., 1973 ; Simpson ef al., 1973 ; Merchant, 1974). Par contre, au cours de la même période, le contenu de l'hypophyse en prolactine diminue (Gros- 
venor ef Turner, 1960 ; Bast ef Melampy, 1972 ; Merchant, 1974 ; Ola et al. 1974). En fin de gestation, les cellules à prolactine révélées dans l'hypophyse par immunocytochimie augmentent en nombre et en taille (El Etréby et Günzel, 1974) ; certaines d'entre elles présentent une réactivité plus grande (Merchant, 1974). Ces observations témoignent d'une activation de la fonction prolactinique de la ratte gestante peu avant le terme.

Chez le fotus de rat et chez le nouveau-né, les données relatives à la fonction prolactinique de l'hypophyse sont à la fois rares et contradictoires. Setalo ef Nakane (1972) ont pu mettre en évidence, par immunocytochimie, des cellules à prolactine dans l'hypophyse fœtale du rat, dès les stades de 16 et 17 jours de gestation. Par contre Watanabe et Daikoku $(1975,1976)$ n'ont pas pu révéler de telles cellules dans des coupes d'hypophyses de fœetus normaux à terme, après traitement avec un antisérum anti-prolactine de rat.

Afin de compléter ces données de la littérature, nous avons d'abord recherché les cellules à prolactine dans des coupes d'hypophyses de rattes gestantes puis de fœtus âgés de 15 à 21 jours, de nouveau-nés et de jeunes jusqu'après le sevrage, en utilisant un anticorps spécifique anti-prolactine de rat ef le complexe peroxydase/ antiperoxidase.

Dans une étude récente, Watanabe et Daikoku $(1975,1976)$ ont observé des cellules à prolactine dans des cultures d'hypophyses fœtales de rat, réalisées à partir de la poche de Rathke isolée à 12 jours de gestation puis cultivée pendant 9 jours pour être examinée à un stade correspondant à 21 jours de gestation. De cette observation, Watanabe et Daikoku ont émis l'hypothèse que les cellules à prolactine peuvent se différencier indépendamment de l'hypothalamus fœetal.

Afin de vérifier in vivo cette hypothèse, nous avons comparé en fin de gestation, l'hypophyse de fœtus normaux à celle de fœus privés chirurgicalement d'hypothalamus par encéphalectomie in utero, à 16 jours.

\section{Techniques expérimentales et matériel utilisé.}

1) Matériel.

Les expériences ont été réalisées sur des rats blancs (Rattus norvegicus albinus) de souche Wistar CF élevés au laboratoire. L'alimentation des animaux se compose de granulés (Labena B, Duquesne-Purina) et d'eau à volonté. Dans les pièces de l'animalerie la température est maintenue à $23^{\circ} \mathrm{C}$ ef l'éclairement est assuré de $6 \mathrm{~h}$ à $20 \mathrm{~h}$.

\section{PLANCHE I}

FIG. 1. - Hypophyse de ratfe ou stade 17 jours $8 \mathrm{~h}$ de la gestation Les cellules à prolactine sont présentes dans la pars distalis (PD) mais absentes dans la pars infermedia $(P I)$. Leur forme est irrégulière $(\times 1000)$.

FIG. 2. - Hypophyse de ratte au stade 21 jours $8 \mathrm{~h}$ de la gestation Dans la pars distalis (PD), les cellules à prolactine sont particulièrement nombreuses $(\times 1000)$. 
Pl. I

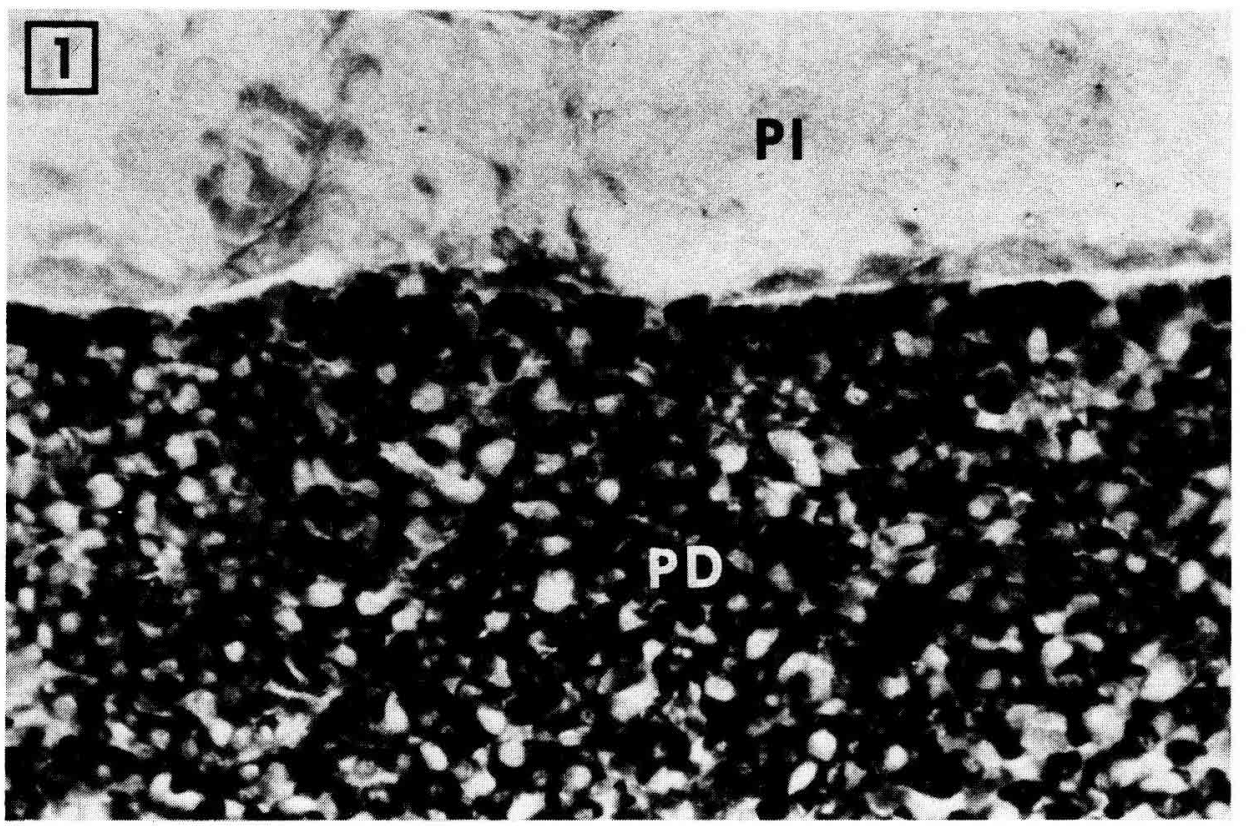

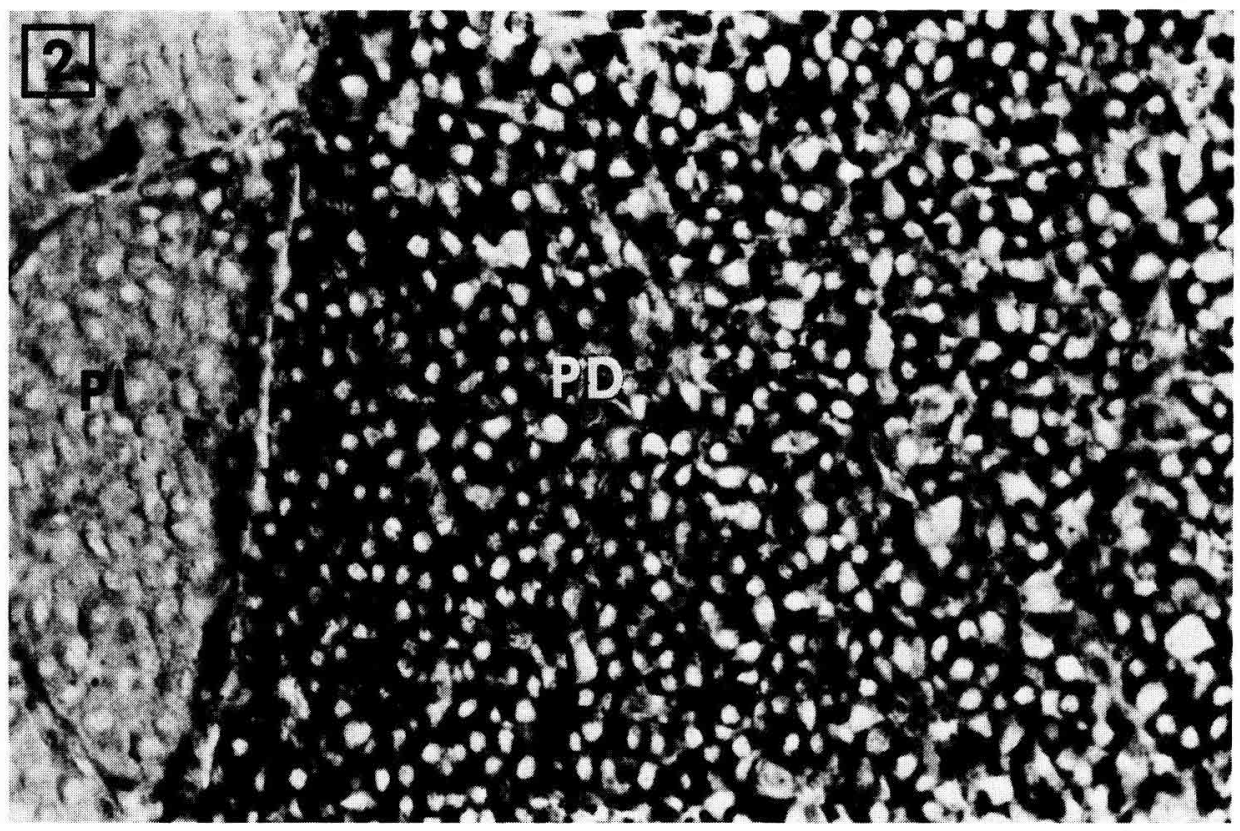


2) Détermination de l'âge des foetus et des jeunes.

La connaissance de l'âge des fœetus nécessite un contrôle précis de la fécondation ; dans ce but, les mâles sont introduits dans les cages des femelles $(1$ mâle pour 6 femelles environ) le soir vers $18 \mathrm{~h}$ et retirés le lendemain matin vers $8 \mathrm{~h}$. On fait alors sur chaque femelle un frottis vaginal ef toutes celles qui présentent des spermatozoïdes dans le frottis, sont présumées pleines et isolées.

L'âge des foetus est compté à partir de l'heure présumée de l'ovulation (premier jour de la gestation) soit environ au milieu de la période de mise au mâle ( $1 \mathrm{~h}$ du matin) (Jost ef Picon, 1970). A titre d'exemple nous préciserons qu'un fœetus prélevé le matin à $9 \mathrm{~h}$ dans le $21 \mathrm{e}$ jour de la gestation est effectivement âgé de 20 jours $8 \mathrm{~h}$; on a coutume de parler du stade de 20 jours 8 h pour la gestation.

Le premier matin qui suit la parturition est le jour 0 post-partum. L'âge des jeunes est compté en jours entiers à partir du jour 0 .

\section{3) Opérations et sacrifices.}

Les animaux ont toujours été sacrifiés le matin vers $9 \mathrm{~h}$ à tous les stades de la gestation, entre 15 et 21 jours ou à différents stades après la naissance : $1,2,3,4,6,8$, $19,20,22,25$ et 30 jours. Le sevrage a eu lieu à 21 jours post-parfum. Quelques femelles gestantes ont été laparotomisées à 16 jours sous anesthésie à l'éther ef 1 à 2 fotus par corne utérine ont été encéphalectomisés selon la technique décrite par Jost (1966) et Jost et al. (1966). Les fołus encéphalectomisés et les témoins des mêmes portées ont été sacrifiés à 21 jours $8 \mathrm{~h}$.

\section{4) Efude immunocytochimique.}

Les hypophyses maternelles, les têtes des fœetus et des jeunes de 1 à 6 jours ou les hypophyses des jeunes âgés de plus de 8 jours ont été fixées $(24 \mathrm{~h})$ par le mélange de Stieve (chlorure mercurique saturé : formol neutre : acide acétique glacial, $75: 20$ : $5 \mathrm{~V} / \mathrm{V}$ ). Elles ont été déshydratées et incluses dans la paraffine.

Les réactions immunocytochimiques ont été réalisées sur des coupes de $7 \mu$ d'épaisseur, selon la technique de Moriarty ef Halmi (1972). Le sérum spécifique antiprolactine de rat a été préparé chez le Lapin et testé par $M$. P. Dubois ; il a été utilisé au $1 / 40$ e. Le complexe PAP (peroxydase-antiperoxydase) a également été préparé chez le Lapin par M. P. Dubois ; il a été utilisé au 1/100.

\section{PLANCHE \|}

FIG. 3. - Partie postérieure d'une hypophyse de fœtus témoin de 21 jours $8 \mathrm{~h}$ Les cellules à prolactine, absentes dans la pars infermedia (PI), sont relativement rares dans la pars distalis (PD). Elles sont plus nombreuses au voisinage de la fente hypophysaire $(\mathrm{FH})(\times 1000)$.

FIG. 4. - Hypophyse de fœetus de 21 jours 8 h, encéphalectomisé à 16 jours

Quelques cellules à prolactine sont bien visibles dans la pars distalis (PD); $\mathrm{PI}=$ pars intermedia ; $\mathrm{FH}=$ fente hypophysaire $(\times 1000)$. 

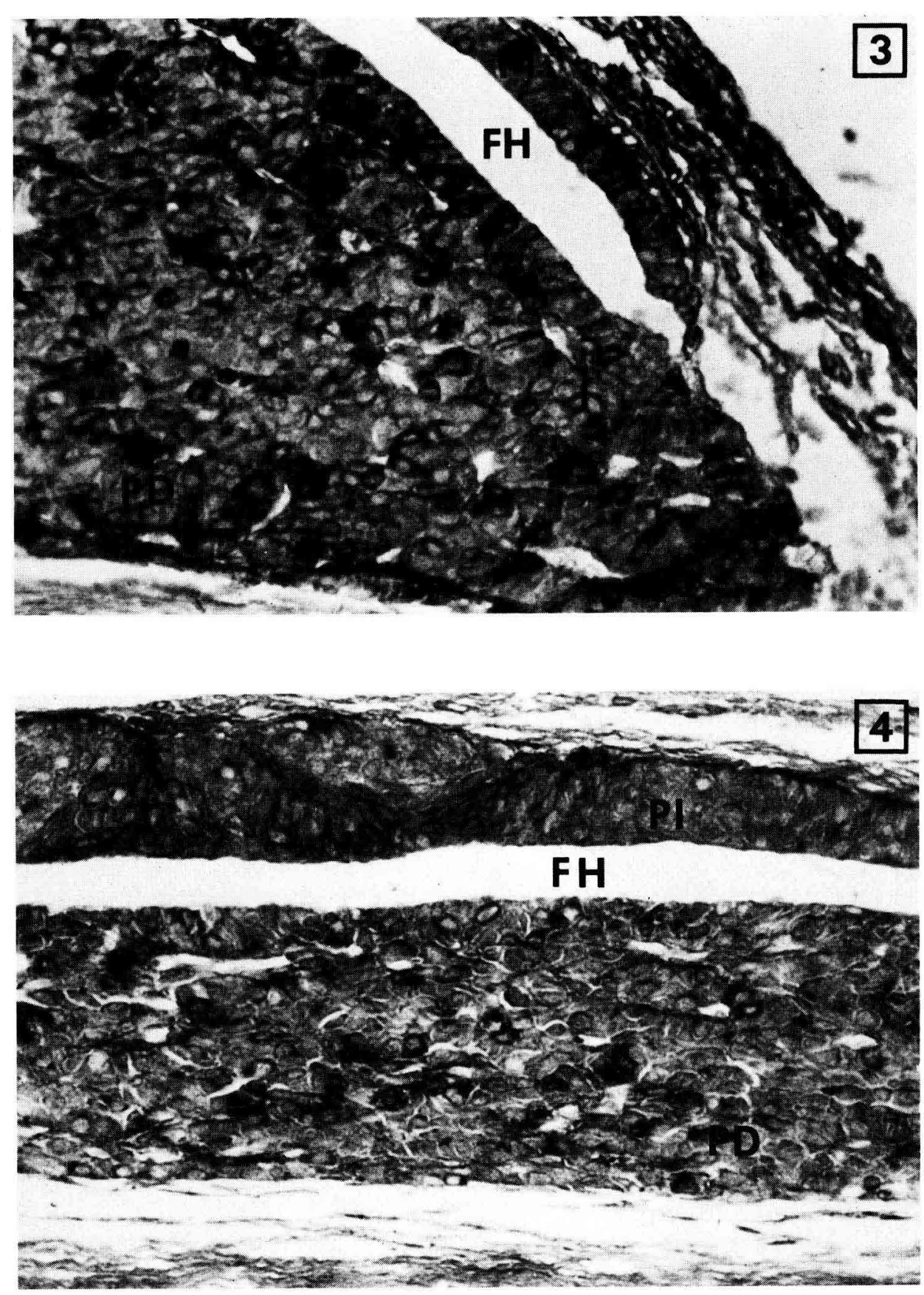
Nous avons employé la 3-3' diaminobenzidine comme substrat de la peroxydase.

Après ces réactions immunocytochimiques, les coupes ont été montées dans le baume du Canada et observées au microscope «Orthomat Leitz » en lumière artificielle.

\section{Résultats.}

1) Cellules à prolactine chez la ratte gestante.

Chez les femelles gestantes, les cellules à prolactine sont très abondantes dans le lobe antérieur de l'hypophyse (fig. 1, 2). Elles présentent une réaction immunocytochimique importante dès le $16 \mathrm{e}$ jour de la gestation. L'intensité de la réaction augmente progressivement pour devenir particulièrement forte à 20 et 21 jours (fig. 2). A ces stades, la réaction est surtout localisée à un pôle de la cellule (fig. 2).

Les cellules à prolactine ont une forme irrégulière (fig. 1, 2) et leur taille augmente légèrement entre 17 et 20 jours.

2) Cellules à prolactine chez les fœtus, les nouveau-nés et les jeunes.

Chez les fœtus, les cellules à prolactine ne sont révélées qu'à partir de 21 jours, le matin, dans la pars distalis (fig. 3). Ces cellules, peu nombreuses, sont localisées près de la fente hypophysaire et dans la moitié postérieure de l'hypophyse. De forme ovoïde ou ronde, ces cellules présentent une très faible réaction.

Chez les nouveau-nés de 1 à 4 jours, l'hypophyse est aussi pauvre en cellules à prolactine que celle des foetus à terme.

Chez les jeunes, c'est à partir du 6 e jour post-parfum que le nombre des cellules à prolactine augmente et que la réaction immunocytochimique devient plus intense.

Dès le $8 \mathrm{e}$ jour, les hypophyses des jeunes mâles contiennent moins de cellules à prolactine que celles des femelles (fig. 5,6).

Au voisinage du $20 \mathrm{e}$ jour après la naissance, $24 \mathrm{~h}$ avant le sevrage, les hypophyses des jeunes animaux des deux sexes sont très riches en cellules à prolactine.

3) Hypothalamus fœetal et différenciation des cellules à prolactine.

L'encéphalectomie des fœtus a été faite à 16 jours, stade de la gestation bien antérieur à celui où l'on peut révéler les premières cellules à prolactine (21 jours).

A terme, dans l'hypophyse des faetus encéphalectomisés, on révèle avec l'antisérum anti-prolactine de rat, autant de cellules à prolactine que dans celle des foetus témoins des mêmes portées (fig. 4).

\section{PLANCHE III}

FIG. 5 et 6. - Hypophyses de jeunes, mâles et femelles âgés de 8 jours Les cellules à prolactine sont plus abondantes chez la femelle que chez le mâle ( $\times 1000)$. 


\section{PI. III}
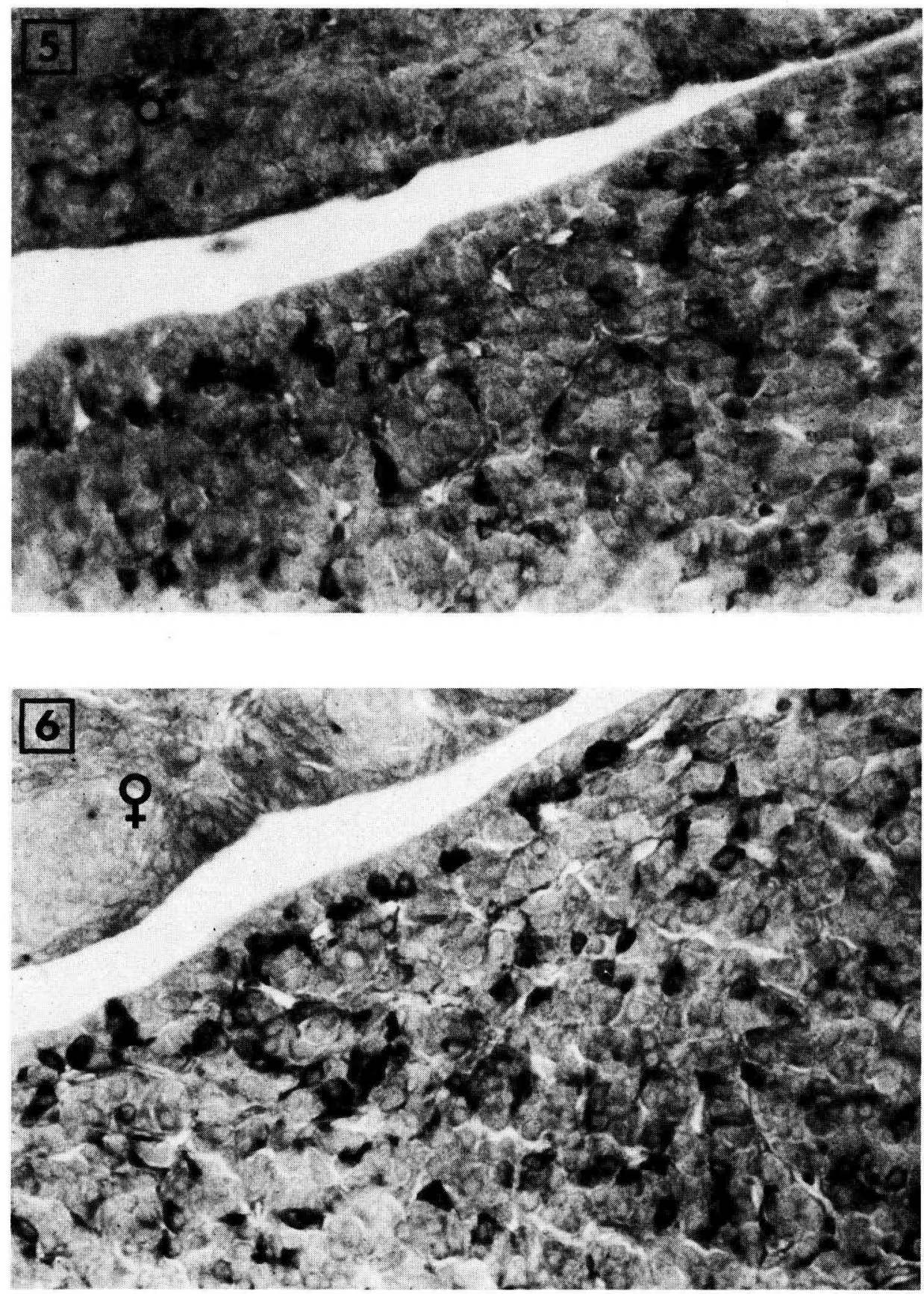


\section{Discussion et conclusions.}

1) Mères: L'évolution des cellules à prolactine dans l'hypophyse maternelle au cours des derniers jours de la gestation est en accord avec les précédentes observations immunocytochimiques de El Etreby et Günzel (1974) et de Merchant (1974). De plus, l'augmentation de l'intensité de la réaction à un pôle de la cellule paraît être le signe d'une polarisation fonctionnelle de la cellule à prolactine comme l'ont observé Vila-Porcile ef al. (1973) en microscopie électronique et non d'une augmentation du contenu cellulaire en hormone puisque, parallèlement, selon plusieurs auteurs, le contenu de l'hypophyse en prolactine diminue. Nos observations cytologiques sont compatibles avec une activation de la fonction prolactinique de la ratte, peu avant le terme, comme en témoigne l'augmentation de la prolactinémie observée par de nombreux auteurs. Cette activation qui survient à quelques heures de la parturition et du début de l'allaitement, pourrait être la conséquence de l'augmentation, en fin de gestation, de la concentration plasmatique en oestrogènes (Yoshinaga ef al., 1969 ; De Lauzon ef al., 1974) associée à la diminution du taux de progestérone (Csapo et Wiest, 1969 ; Wiest, 1970). Chez la Ratte, les œstrogènes ont la faculté de stimuler les cellules à prolactine (El Etreby ef Günzel, 1974) et d'augmenter la synthèse ef la sécrétion de prolactine aussi bien in vivo que in vitro (Yamamoto et al., 1975). Dans les hypophyses de rattes gestantes à terme, injectées quotidiennement de $50 \mu \mathrm{g}$ de benzoate d'œstradiol, du $15^{\mathrm{e}}$ au $21 \mathrm{e}$ jour de la gestation, nous avons observé des cellules à prolactine présentant une faible réaction immunocytochimique ce qui peut suggérer une intense sécrétion.(Chatelain et Dupouy, non publié).

2) Fœtus : Chez le fœtus de Rat, nous avons noté une apparition très tardive des cellules à prolactine puisqu'elles ne sont pas décelables par immunocytochimie avant 21 jours. Nos résultats sont en désaccord d'une part avec ceux de Setalo et Nakane (1972) qui ont observé une apparition bien plus précoce de ces cellules, aux stades de 16 et 17 jours de gestation, et d'autre part avec ceux de Watanabe et Daikoku (1975, 1976) qui n'ont pas réussi à mettre en évidence des cellules à prolactine dans l'hypophyse de foetus à terme.

L'existence de cellules à prolactine dans l'hypophyse de fœetus encéphalectomisés est un argument en faveur d'une non-intervention de l'hypothalamus fœetal dans la différenciation des cellules à prolactine in vivo. On peut rappeler d'ailleurs qu'une différenciation indépendante de l'hypothalamus foetal a été précédemment observée in vivo, aussi bien pour les cellules corticotropes (ACTH) ef mélanotropes (MSH) (Chatelain ef al., 1976) que pour les cellules thyréotropes (TSH) et gonadotropes (FSH/LH) (Dupouy et al., non publié). Les résultats de nos études in vivo sont par ailleurs en parfait accord avec ceux obtenus par Watanabe ef Daikoku $(1975,1976)$ in vitro, à partir de cultures de tissu hypophysaire foetal.

Chez le foetus de Hamster, Thompson et Trimble (1976) ont mis en évidence par immunocytochimie des cellules à prolactine dans la pars distalis, dès le $13 \mathrm{e}$ jour de la gestation ; par la suite, le nombre et la répartition de ces cellules ne varient pas mais quelques cellules à prolactine sont également décelables dans la pars tuberalis, la veille de la parturition qui intervient à 16 jours de gestation.

Chez le fœitus humain, quelques rares cellules à prolactine ont été détectées à 
16 semaines $1 / 2$ de gestation, grâce à un anti-sérum anti-prolactine bovine (Baker et Jaffe, 1975).

3) Nouveau-nés et jeunes : L'augmentation du nombre de cellules à prolactine dans l'hypophyse des nouveau-nés et des jeunes, au cours de la première quinzaine de la vie extra-utérine coïncide avec le développement de l'axe hypothalamo-hypophyso-thyroïdien marqué par une importante élévation des taux de TRH dans l'hypothalamus, de TSH dans l'hypophyse et dans le plasma, d'hormones thyroïdiennes dans le sang (Dussault et Labrie, 1975). On sait par ailleurs que les hormones thyroĩdiennes peuvent influencer la sécrétion de prolactine chez l'adulte. II reste à déterminer si les hormones de l'axe hypothalamo-hypophyso-thyroïdien sont susceptibles de jover un rôle dans le processus de différenciation des cellules à prolactine.

Chez le Hamster, au cours des 3 premiers jours post-parfum, Thompson et Trimple (1976) ont noté une augmentation du nombre de cellules à prolactine mais celles-ci présentent une réactivifé à l'anti-sérum très inférieure à celle de la femelle adulte.

Ces observations indiquent que, chez le Rat comme chez le Hamster, la différenciation des cellules à prolactine débute avant la naissance et se poursuit au cours des premiers jours de la vie extra-utérine.

8e Colloque de Neuroendocrinologie. - Table ronde Prolactine. Tours, 7 septembre 1976.

\section{Références}

AMENOMORI Y., CHEN C. L., MEITES J., 1970. Serum prolactin levels in rats during different reproductive states. Endocrinology, 86, 506-510.

BAKER B. L., JAFFE R. B., 1975. The genesis of cell types in the adenohypophysis of the human fetus as observed with immunocytochemistry. Amer. J. Anat., 143, 137-162.

BAST J. D., MELAMPY R. M., 1972. Luteinizing hormone, prolactin and ovarian $20 \alpha$ hydroxysteroid dehydrogenase levels during pregnancy and pseudopregnancy in the rat. Endocrinology, 91, 1499-1505.

CHATELAIN A., DUBOIS M. P., DUPOUY J. P., 1976. Hypothalamus and cytodifferentiation of the fœial pituitary gland. Cell Tiss. Res., 169, 335-344.

CHEN C. L., MEITES J., 1970. Effects of estrogen and progesterone on serum and pituitary prolactin levels in ovariectomized rats. Endocrinology, 86, 503-505.

CSAPO A. I., WIEST W. G., 1969. An examination of the quantitative relationship between progesterone and the maintenance of pregnancy. Endocrinology, 85, 735-746.

DE LAUZON S., UHRICH F.; VANDEL S., CITTANOVA N., JAYLE M. F., 1974. Determination of progesterone and of free and conjugated estrogens in pregnant and pseudo-pregnant rats. Steroids, 24, 31-40.

DUSSAULT J. H., LABRIE F., 1975. Development of the hypothalamic-pituitary-thyroid axis in the neonatal rat. Endocrinology, 97, 1321-1324.

EL ETREBY M. F., GÜNZEL P., 1974. Sex hormones-effects on prolactin cells in the rat, dog, monkey and man. Acta endocr., 76, suppl. 189, 3-15.

GROSVENOR C. E., TURNER C. W., 1960. Pituitary lactogenic hormone concentration during pregnancy in the rat. Endocrinology, 66, 96-99.

JOST A., 1966. Anterior pituitary function in fœtal life. In G. W. HARRIS, B. T. DONOVAN, The pifuitary gland, vol. 2, 299-323, Butterworths, Londres.

JOST A., DUPOUY J. P., MONCHAMP A., 1966. Fonction corticotrope de l'hypophyse ef hypothalamus chez le foetus de rat. C. R. Acad. Sci. Paris, 262, 147-150. 
JOST A., PICON L. O., 1970. Hormonal control of fetal development and metabolism. Adv. metab. disord. 4, 123-184.

LINKIE D. M., NISWENDER G. D., 1972. Serum levels of prolactin luteinizing hormone, and follicle stimulating hormone during pregnancy in the rat. Endocrinology, 90, 632-637.

MERCHANT F. W., 1974. Prolactin and luteinizing hormone cells of pregnant and lactating rats as studied by immunohistochemistry and radioimmunoassay. Am. J. Anat., 139, 245-268.

MORIARTY G. C., HALMI N. S., 1972. Electron microscopic study of the adrenocorticotropin-producing cell with the use of unlabeled antibody and the soluble peroxidase-antiperoxidase complex. J. histochem. cytochem., 20, 590-603.

MORISHIGE W. K., PEPE G. J., ROTHCHILD I., 1973. Serum lufeinizing hormone, prolactin and progesterone levels during pregnancy in the rat. Endocrinology, 92, 1527-1530.

NAGASAWA H., YANAI R., 1972. Changes in serum prolactin levels shortly before and after parturition in rats. Endocr. jap., 19, 139-143.

OTA K., OTA T., YOKOYAMA A., 1974. Plasma corticosterone concentrations and pituitary prolactin content in late pregnancy and their within-day fluctuations in the rat. J. Endocr., 61, 21-28.

SETALO G., NAKANE P. K., 1972. Studies on the functional differentiation of cells in fetal anterior pituitary glands of rats with peroxidase-labelled antibody method. Anat. Rec., 172, 403-404.

SIMPSON A. A., SIMPSON M. H. W., SINHA Y. N., SCHMIDT G. H., 1973. Changes in concentration of prolactin and adrenal corticosteroids in rat plasma during pregnancy and lactation. J. Endocr., 58, 675-676.

SHIU R. P. C., KELLY P. A., FRIESEN H. G., 1973. Radioreceptor assay for prolactin and other lactogenic hormones. Science, 180, 968-971.

THOMPSON S. A., TRIMBLE J. J., 1976. Immunohistochemical localization of prolactin cells of the pars distalis in the fetal and neonatal hamster. Cell Tiss. Res., 168, 161-175.

VILA-PORCILE E., OLIVIER L., RACADOT J., 1973. L'architecture ultrastructurale du cordon adénohypophysaire et la polarisation fonctionnelle des cellules : cas de la cellule à prolactine chez la ratte. 5e colloque de Neuroendocrinologie, Marseille, 13-14 septembre 1973.

WATANABE Y. G., DAIKOKU S., 1975. Immunocytological study on the cytodifferentiation of rat anterior pituitary in vivo and in vitro. 1Dth. Int. Cong. Anat., Tokyo, p. 275.

WATANABE Y. G., DAIKOKU, 1976. Immunohistochemical study on adenohypophysial primordia in organ culture. Cell Tiss. Res., 166, 407-412.

WIEST W. G., 1970. Progesterone and $20 \alpha$ hydroxypregn 4-en 3-one in plasma, ovaries and uteri during pregnancy in the rat. Endocrinology, 87, 43-48.

YAMAMOTO K., KASAI K., IEIRI T., 1975. Control of pituitary functions of synthesis and release of prolactin and growth hormone by gonadal steroids in female and male rats. Jap. J. Physiol., 25, 645-648.

YOSHINAGA K., HAWKINS R. A., STOCKER J. F., 1969. Estrogen secreti on by the rat ovary in vivo during the estrous cycle and pregnancy. Endocrinology, 85, 103-112. 